\title{
Review of "Definition"
}

\author{
Franz Plochberger
}

It's nearly philosophically to define the word Definition as term. If you define a mathematical, numeric rule it's clear. On the other side you have a nearly uncountable amount of genuine verbal possibilities to fix a Definition.

It seems to be important to find a wide form by a few simple words. Too much words may minimize your goal. 